\title{
Prevalence and Impact of Early Prone Position on 30-day Mortality in Mechanically Ventilated Patients With COVID-19 - a Nationwide Cohort Study
}

\section{Lars Engerström}

Linköping University

Johan Thermaenius

Karolinska Institute

Johan Mårtensson

Karolinska Institute

Anders Oldner

Karolinska Institute

Johan Petersson

Karolinska Institute

Jessica Kåhlin

Karolinska Institute

Emma Larsson ( $\nabla$ emma.larsson@ki.se )

Karolinska Institute

\section{Research Article}

Keywords: Prone position, critical illness, covid-19, ventilation, outcome, mortality, ARDS

Posted Date: February 1st, 2022

DOI: https://doi.org/10.21203/rs.3.rs-1305613/v1

License: (c) (i) This work is licensed under a Creative Commons Attribution 4.0 International License.

Read Full License 


\section{Abstract}

Background: COVID-19 ARDS shares features with non-COVID ARDS but also demonstrates distinct physiological differences. Despite a lack of strong evidence, prone positioning has been advocated as a key therapy for COVID-19 ARDS. The effects of prone position in critically ill patients with COVID-19 are not fully understood, nor is the optimal time of initiation defined. In this nationwide cohort study, we aimed to investigate the association between early initiation of prone position and mortality in mechanically ventilated COVID-19 patients with low oxygenation on ICU admission.

Methods: Using the Swedish Intensive Care Registry (SIR), all Swedish ICU patients $\geq 18$ years of age with COVID-19 admitted between March 2020, and April 2021 were identified. A sub-cohort of patients with $\mathrm{PaO} 2 / \mathrm{FiO} 2$ ratio $\leq 20 \mathrm{kPa}$ on ICU admission and receiving invasive mechanical ventilation within 24 hours from ICU admission was generated. In this sub-cohort the association between early use of prone position (within 24 hours from intubation) and 30-day mortality was estimated using univariate and multivariable logistic regression models.

Results: The total study cohort included 6350 ICU patients with COVID-19, of whom $46.4 \%$ were treated with prone position ventilation. Overall 30-day mortality was $24.3 \%$. In the sub-cohort of 1714 patients with lower admission oxygenation, the utilization of early prone increased from $8.5 \%$ in March 2020 to $48.1 \%$ in April 2021. The crude 30 -day mortality was $27.2 \%$ compared to $30.2 \%$ in patients not receiving early prone positioning. We found no significant association between early use of prone positioning and survival.

Conclusions: During the first three waves of the COVID-19 pandemic, almost half of the patients in Sweden were treated with prone position ventilation. We found no association between early use of prone positioning and survival in patients on mechanical ventilation with severe hypoxemia on ICU admission. To fully elucidate the effect and timing of prone position ventilation in critically ill patients with COVID-19 further studies are desirable.

\section{Introduction}

The current Coronavirus disease (COVID-19) pandemic has affected healthcare worldwide with large numbers of critically ill patients, where the surge of patients often outnumbered intensive care unit (ICU) resources. SARS-CoV-2 induces a large variation of symptoms, from mild catarrhalia to severe acute respiratory distress syndrome (ARDS) with requirement for invasive mechanical ventilation. Early prone position ventilation is an established intervention in patients with ARDS (1) leading to improved ventilation-perfusion matching and survival. Even so, prone position continues to be underused in moderate to severe ARDS $(2,3)$. Recent studies during the COVID-19 pandemic indicate a striking increase in the use of prone position, despite scarce ICU resources $(4,5)$.

Interestingly, observational studies have found an association between the oxygenation response to prone positioning and improved outcome for COVID-19 ARDS $(6,7)$. This contradicts previous findings in 
patients with non-COVID-19 ARDS where such an association is missing (8). ARDS caused by COVID-19, as opposed to other etiologies, is reported to be more severe, display a lower $\mathrm{PaO} / \mathrm{FiO} 2$ ratio but with a greater lung compliance (6). However, other studies reported a similar compliance in COVID-19 and nonCOVID-19 ARDS (9). Despite a lack of strong outcome evidence in COVID-19 ARDS prone positioning has been advocated as a key therapy in COVID-19 ARDS $(5,10-12)$.

The beneficial physiological effects of prone position with improved ventilation-perfusion matching and more homogenous inflation which may lead to less stress and strain and reduced inspiratory pressures are well established, but there is lack of evidence of whether this results in an overall survival improvement in invasively mechanically ventilated patients with COVID-19.

Few population-based reports have described the utilization of prone position in critically ill patients with COVID-19. Previous studies primarily describe the first wave of COVID-19 and with conflicting results on mortality benefit from early prone position (13-15). Thus, uncertainty prevails regarding the association between prone position, including time of initiation, and outcome. In this nationwide cohort study, we aimed to investigate the use of prone position ventilation and to evaluate the overall effect on mortality of early initiation (within 24 hours from intubation) of prone position in a sub-cohort of mechanically ventilated COVID-19 patients with a PaO2/FiO2 ratio $\leq 20 \mathrm{kPa}$ on admission.

\section{Material And Methods}

The Swedish Ethical Review Authority approved the study (approval number 2020-01477) and waived requirement for informed consent. The study adhered to the STROBE (Strengthening the Reporting of Observational Studies in Epidemiology) guidelines for cohort studies (16). All research was conducted in accordance with national guidelines and regulations.

\section{Study design and population}

All public health care in Sweden, including intensive care, is tax-funded and available for all citizens regardless of private health insurances. In co-operation with the Public Health Agency of Sweden, mandatory surveillance data of COVID-19 are routinely reported to the Swedish Intensive Care Registry (SIR). SIR is collecting individual patient data within the legal framework of the Swedish National Quality Registries. Written informed consent is not required, but patients have the possibility to withdraw their data from SIR at any time. Available data include baseline demographics, comorbidities, variables included in the Simplified Acute Physiology Score (SAPS3) and variables on process of care in the ICU. Data are transferred to SIR after local validation. After central validation at SIR, incomplete or inconsistent (entries outside pre-specified limits) data are returned to the specific ICUs for correction before data are added to the SIR database. All Swedish citizens have a unique personal identity number making linkage possible to the Swedish Population Register, and thereby ascertain mortality data. The personal identity number also enables analyses of readmissions and to follow the care of a patient between different ICUs. 
In this nationwide cohort study, we identified all Swedish ICU patients $\geq 18$ years of age with confirmed SARS-CoV-2 by polymerase chain reaction admitted between March 6, 2020, and April 30, 2021. Exclusion criteria included SARS-CoV-2-RNA positive patients with other reason for admission than COVID-19 and missing data on follow up (due to temporary personal identity number).

For the analyses of the impact of early (within 24 hours from intubation) initiation of prone position on 30-day mortality, a sub-cohort was extracted with the following inclusion criteria: patients with a $\mathrm{PaO} 2 / \mathrm{FiO} 2$ ratio $\leq 20 \mathrm{kPa}$ within one hour before until one hour after arrival to ICU (registered for SAPS3 calculation) and receiving invasive mechanical ventilation within 24 hours from ICU admission.

\section{Covariates and outcome}

Baseline characteristics, including comorbidities, were defined at the time of ICU admission. Physiological variables were recorded on admission within one hour before until one hour after arrival to ICU. Within this time interval, for each parameter the worst was included in the analyses. Information on process of care includes the entire ICU stay. Thirty-and 90-day mortality were defined as mortality (all-cause) within 30 and 90 days from admission to ICU, respectively. Primary outcome was 30-day mortality and secondary outcome was 90-day mortality.

\section{Statistical analysis}

Categorical variables are presented as number with percentage. Continuous variables are presented as median with interquartile range (IQR).

For patients with a $\mathrm{PaO} 2 / \mathrm{FiO} 2$ ratio $\leq 20 \mathrm{kPa}$ on $\mathrm{ICU}$ admission and receiving invasive mechanical ventilation within 24 hours from ICU admission, the association between early use of prone position (within 24 hours from intubation) and 30-day mortality was estimated using univariate and multivariable logistic regression models and expressed as odds ratios (OR) with corresponding $95 \%$ confidence intervals. A priori selected covariates including patient sex, age, comorbidities (cardiac disease, chronic obstructive pulmonary disease (COPD)/asthma, obesity (BMl $>40 \mathrm{~kg} / \mathrm{m} 2$ ), hypertension, immune deficiency, chronic liver disease, chronic kidney disease, neuromuscular disease and malignancy (neoplasia spread beyond regional lymph nodes)), PaO2/FiO2 ratio, SAPS3 and admission period. To avoid collinearity, age, comorbidity and $\mathrm{PaO} 2 / \mathrm{FiO} 2$ ratio components were removed from SAPS3. In addition, we performed a logistic regression model exploring 90-day mortality. All variables in the univariate models were carried forward to the multivariable models. To test the robustness of our findings we performed logistic regression models exploring 30-day mortality in the following two subgroups 1) patients with $\mathrm{PaO} 2 / \mathrm{FiO} 2$ ratio $\leq 13.3 \mathrm{kPa}$ on ICU admission and 2) patients still in treated in ICU within 48 hours from ICU admission.

Data were analysed as complete cases. A p-value of 0.05 was considered statistically significant. All data were analysed using R 4.1.1 (R Core Team (2021). R Foundation for Statistical Computing, Vienna, Austria) and Stata/SE 16 (StataCorp, Collage Station, TX, USA). 


\section{Results}

\section{Patients}

From March 6, 2020, to April 30, 2021, a total of 7063 ICU patients with confirmed SARS-CoV-2 were reported to SIR. We excluded 574 patients with a primary diagnosis not associated with COVID-19 and 139 patients without data on follow-up (patients who are not Swedish residents and receive temporary patient numbers and patients emigrating during the study), yielding a total study cohort of $6350 \mathrm{ICU}$ patients with COVID-19 (figure 1).

Approximately half of the patients $(46.4 \%)$ were treated with prone position ventilation (table 1 ).

Patients in the total study cohort never receiving prone positions ventilation during ICU stay are hereafter referred to as "prone=no" and patients receiving prone position as "prone=yes".

From the total study cohort, we extracted a sub-cohort of patients with $\mathrm{PaO} 2 / \mathrm{FiO} 2$ ratio $\leq 20 \mathrm{kPa}$ on ICU admission and receiving invasive mechanical ventilation within 24 hours from ICU admission. To generate this sub-cohort the following patients were excluded from the total study cohort: 1978 patients with no data on $\mathrm{PaO} 2 / \mathrm{FiO} 2$ ratio on ICU admission, 568 patients with $\mathrm{PaO} 2 / \mathrm{FiO} 2>20 \mathrm{kPa}$ on ICU admission, 1877 patients who did not received invasive mechanical ventilation within 24 hours from ICU admission, 57 patients with no information on proning and 156 patients with no information on time of start of proning (Figure 2).

Thus, 1714 ICU patients were identified in this sub-cohort and included in the logistic regression models of 30- and 90-day mortality. Patients within this sub-cohort who were not treated with prone position initiated within 24 hours from start of invasive mechanical ventilation are hereafter referred to as "early prone $=$ no" and patients treated with prone position within 24 hours from start of invasive mechanical ventilation as "early prone $=$ yes".

\section{Total study cohort - baseline characteristics and process of care}

Of the total cohort 6350 patients, 1843 (29.0\%) were women. Median age was 64 (IQR 55-72) years. For $393(6.2 \%)$ of the patients there were no data on proning. Most of the patients were admitted from hospital floor (77.6\%). Median duration of symptoms before ICU admission was 10 (IQR 7-13) days. Approximately one quarter $(27.8 \%)$ of the patients had no reported comorbidity on admission. Baseline characteristics are presented in detail in supplementary table 1.

Median total length of ICU stay was 10 (IQR 4-19) days; 5 (IQR 2-11) days for "prone=no" and 15 (IQR 926) days for "prone=yes". Overall, 4174 patients (65.7\%) patients received invasive mechanical ventilation. The median total duration of invasive mechanical ventilation was 11.6 (IQR 6.2-20.4) days. The median time from ICU-admission to start of invasive mechanical ventilation was 4.0 hours and the median time from ICU-admission to treatment in prone position was 20.4 hours. Renal replacement therapy was reported in 771 (13.5\%) of the patients, 55 (1.6\%) patients received extra corporeal 
membrane oxygenation (ECMO) and 1562 (24.6\%) patients underwent tracheostomy. Care provided in the ICU for the total study cohort is presented in supplementary table 2.

Patients with PaO2/FiO2 ratio $\leq 20 \mathrm{kPa}$ on ICU admission and receiving invasive mechanical ventilation within 24 hours from ICU admission - baseline characteristics and process of care

Baseline characteristics are presented in table 1. Of the 1714 patients, $512(29.9 \%)$ were women and the median age was 64 (IQR 55-71) years. Approximately one-third (28.8\%) had no reported comorbidity on admission; 356 (31.1\%) and 137 (24.0\%) for "early prone=no" and "early prone=yes", respectively. Median $\mathrm{PaO} 2 / \mathrm{FiO} 2$ ratio on ICU admission was 11.6 (IQR 8.8-14.8) kPa for "early prone=no" and 9.9 (IQR 8.012.6) $\mathrm{kPa}$ for "early prone=yes". For "early prone=no", median SAPS 3 score was 58 (IQR 53-65) and "early prone=yes" was 58 (IQR 53-66).

The use of early prone increased from $8.5 \%$ in March 2020 to 48.1\% in April 2021. The median total duration of invasive mechanical ventilation was 11.0 (IQR 5.7-18.8) days for patients with "early prone = no" and 11.2 (6.9-18.5) days for patients with "early prone = yes". Renal replacement therapy was reported in $20.2 \%$ and $16.7 \%$ for "early prone=no" and "early prone=yes", respectively. Median total length of ICU stay was 14 (IQR 8-22) days for "early prone=no" and 14 (IQR 9-22) days for "early prone=yes". Care provided in the ICU for the sub-cohort is presented in table 2.

\section{Mortality}

Overall, 30-day mortality was $24.3 \%, 22.3 \%$ and $26.4 \%$ for "prone=no" and "prone=yes", respectively. For patients with no information on proning 30 -day mortality was $23.2 \%$. Corresponding figures for 90 -day mortality were, $29.0 \%, 24.3,33.6$, and 28.8 (supplementary table 1 ).

For patients with $\mathrm{PaO} 2 / \mathrm{FiO} 2$ ratio $\leq 20 \mathrm{kPa}$ on ICU admission and receiving invasive mechanical ventilation within 24 hours from ICU admission, 30-day mortality was $29.2 \%, 30.2 \%$ and $27.2 \%$ for "early prone=no and "early prone=yes" respectively. The corresponding figures for 90 -day mortality were $33.5 \%$, $34.8 \%$ and $31.1 \%$ (table 1. )

As shown in Figure 2, 1978 (31.1\%) patients had missing data on PaO2/FiO2 ratio on ICU admission. A majority of these patients did not receive mechanical ventilation on admission, and under these circumstances $\mathrm{FiO} 2$ is not routinely reported to SIR. The median age among these patients was 64 (IQR 54-72) years, $542(27.4 \%)$ were women and 393 (19.8\%) patients had died within 30 days from ICU admission.

Logistic regression estimating the association between initiation of early prone position ventilation and mortality

The regression analyses included only patients with $\mathrm{PaO} 2 / \mathrm{FiO} 2$ ratio $\leq 20 \mathrm{kPa}$ on ICU admission and receiving invasive mechanical ventilation within 24 hours from ICU admission. On univariate logistic regression analysis of 30-day mortality, the odds ratio for "early prone=yes" compared to "early prone=no" 
was 0.86 (95\% $\mathrm{Cl} 0.69-1.08)$, and after adjustment the corresponding odds ratio was 0.92 (0.71-1.19) (Table 3).

The odds ratios remained somewhat unchanged in the analyses restricted to patients with a) $\mathrm{PaO} 2 / \mathrm{FiO} 2$ ratio $\leq 13.3 \mathrm{kPa}$ on ICU admission and $b$ ) patients still in treated in ICU within 48 hours from ICU admission, Supplementary Table 3 and 4 . We also performed a logistic regression model of 90-day mortality with almost identical results, Supplementary Table 5.

\section{Discussion}

In this nationwide study we describe the utilization of prone position in more than 6000 critically ill patient with COVID-19, where approximately half of the patients received prone position ventilation. Using a sub-cohort of 1714 mechanically ventilated patients with respiratory insufficiency and hypoxemia we investigated the impact of early initiation of prone position on mortality. We found no association between early proning and survival. The crude 30 -day mortality was $27.2 \%$ compared with $30.2 \%$ in patients not receiving early prone positioning.

After the striking results of the PROSEVA-study, recommended standard management of ARDS comprises of low tidal volumes, plateau pressures $<30 \mathrm{~cm} \mathrm{H} 2 \mathrm{O}$ and prone ventilation for moderate and severe ARDS with $\mathrm{PaO} 2 / \mathrm{FiO} 2<13.3 \mathrm{kPa}(10)$. Adherence to an ARDS-protocol based on these parameters has been associated with improved survival (17). Nevertheless, prone ventilation has been reported to be markedly underused with only $16.3 \%$ proning of severe ARDS and $5.5 \%$ of moderate ARDS patients in the LUNGSAFE study (3) and $32.9 \%$ and $10.3 \%$ for severe and moderate ARDS, respectively in the ARDS Prone Position Network (APRONET) study published two years later (2). The most prominent reason for refraining prone ventilation was the apprehension that the oxygenation was not impaired enough or improving. Additional explanations included hemodynamic instability, deficient availability of competent staff and expected increase in workload (2).

During the COVID-19 pandemic the use of prone position for ARDS patients has increased considerably with a reported frequency ranging from $30 \%$ up to $75 \%(4,13,14)$. This may reflect the limited treatment options for COVID-19 as well as increased adherence to current guidelines when hospitals were challenged with an overload of ARDS-patients. The use of prone position in this Swedish cohort is comparable to previous COVID-19 related ARDS studies; in total $47.3 \%$.

In the current study, early initiation of prone position in a subgroup of mechanically ventilated patients with $\mathrm{PaO} 2 / \mathrm{FiO} 2 \leq 20 \mathrm{kPa}$ had lower crude 30-day mortality compared to patients not receiving early initiation of prone position ventilation. This difference did not, however, reach statistical significance. Previous studies are relatively few and report varying results. In a study by Mathews et al, an increased 60-day survival was demonstrated in patients treated with prone ventilation within two days of ICU admission during the first wave of COVID-19 in the US (14). Furthermore, a systematic review and metaanalysis on prone versus supine ventilation in COVID-19 patients reported an improved PaO2/FiO2 ratio with prone position and a lower mortality in patients that had been ventilated in prone position, 
although many small studies in the metaanalysis renders a low level of evidence level (18). In addition, the Italian ICU-RER COVID-19 Collaboration demonstrated a correlation between oxygenation improvement due to prone positioning and ventilator-free days at 28 days and that non-responders had an increased mortality compared to responders(7). On the contrary, an Italian multi-center study from the first COVID-19 wave with a high utilization of prone positioning could not demonstrate a survival benefit, rather a worse outcome mainly attributed to the higher disease severity in the proning group (13).

There are several possible explanatory factors to differences in previous results and the current study. In our study we only had data on $\mathrm{PaO} 2 / \mathrm{FiO} 2$ ratio on ICU admission, thus we could not follow the dynamics in the severity of the patient condition after start of intensive care. Various definitions and modeling of admission period as a variable in analyses could also bias the results as optimal management of this variable is not clear. We included the admission period as a possible confounder in the analyses as both mortality and utilization of prone position have varied during the pandemic. In addition, and very important to point out, our study only investigates the effect of initiation of early prone position ventilation. Thus, patients in the group "early prone=no" could be treated with prone position after 24 hours from start of invasive mechanical ventilation. To fully elucidate the impact of prone position ventilation in critically ill patients with COVID-19 a randomized controlled trial would be desirable. However, in consideration of the positive results from the PROSEVA trial, a randomized trial could be disputable from an ethical perspective.

As in previous studies on COVID-19, the predominant comorbidities were chronic hypertension, diabetes mellitus, COPD, cardiac disease and obesity $(5,19)$. Most patients were admitted to the ICU from another hospital floor rather than the emergency department. A greater proportion of patients experienced severe ARDS as compared to previous studies, largely explained by the inclusion criteria of PaO2/FiO2 $20 \mathrm{kPa}$. The overall mortality of $29.5 \%$ in the cohort hence reflects a critically ill group of predominantly severe ARDS patients.

Our study has several strengths. We analyzed a large, nationwide multicenter cohort of ICU patients with COVID-19 with almost complete follow-up. All COVID-19 ICU patients in Sweden were eligible for inclusion, providing high generalizability to similar health-care systems. In addition, the inclusion period covered three peaks of the pandemic. SIR includes data on pre-ICU comorbid conditions, process of ICU care and long-term follow-up. Follow-up is ascertained by linkage between SIR and national registries. Furthermore, data in SIR are prospectively reported for quality-surveillance purposes and are therefore unbiased in relation to this study.

We also identify several limitations, including the registry-based study design. We had no data on socioeconomic status nor ethnicity. Data on process of care before ICU admission are not included in SIR. Approximately one-third (31.1\%) of the total study cohort had no data on $\mathrm{PaO} 2 / \mathrm{FiO} 2$ ratio on ICU admission. A majority of these patients did not receive any non-invasive or invasive mechanical ventilation on admission and 30-day mortality was $19.8 \%$ compared with $29.2 \%$ for patients with $\mathrm{PaO} 2 / \mathrm{FiO} 2$ ratio $\leq 20 \mathrm{kPa}$ on ICU admission and receiving invasive mechanical ventilation within 24 hours 
from ICU admission. Information on ventilator settings, duration and the number of proning sessions and contraindications to prone position would have added value to this study, but these data were unfortunately not available, nor was data on the dynamics of $\mathrm{PaO} 2 / \mathrm{FiO} 2$ ratio. Furthermore, due to the rapid and persistent increase in the need of ICU beds, ICU recourses were of course strained during the study period. The global cumulative knowledge concerning treatment of COVID-19 and treatment algorithms are constantly evolving. Treatment with high-dose low molecular weight heparins is not included in SIR, nor is the use of steroids. Finally, it is possible that the registration of prone position in SIR in some cases also could include patients only partially proned i.e., placed in the side position.

\section{Conclusions}

In this nationwide cohort study of more than 6000 ICU COVID-19 patients covering several pandemic peaks, almost half of the patients were treated with prone position ventilation. The use of early prone position treatment increased markedly over time in patients with severe hypoxemia. We found no association between early use of prone positioning and survival in patients on mechanical ventilation with severe hypoxemia on ICU admission. The crude 30 -day mortality was $27.2 \%$ compared to $30.2 \%$ in patients not receiving early prone positioning. To fully elucidate the effect and timing of prone position ventilation in critically ill patients with COVID-19 further studies are desirable.

\section{Declarations}

\section{Ethics approval}

This study was approved by the Swedish Ethical Review Authority (approval number 2020-01477). SIR collects individual data from all Swedish ICUs and operates within the legal framework of the Swedish National Quality Registries. This framework does not require written informed consent from the patients, but patients may withdraw their data from the registry at any time.

\section{Consent for publication}

Not applicable.

\section{Availability for data and material}

The data that support the findings of this study are available from SIR but restrictions apply to the availability of these data, which were used under license for the current study, and so are not publicly available. Data are however available from the authors upon reasonable request and with permission of SIR.

\section{Competing interests}

The authors report no conflicts of interest. 


\section{Funding}

The study received sponsorship from The Knut and Alice Wallenberg Foundation. The Knut and Alice Wallenberg Foundation had no role in design and conduct of the study; collection, management, analysis, and interpretation of the data; preparation, review, or approval of the manuscript; and decision to submit the manuscript for publication.

\section{Author contributions}

LE, JK and EL had full access to the data in the study and take responsibility for the integrity of the data, the accuracy of the data analysis and the decision to submit for publication.

Concept and design: LE, JK and EL

Acquisition, analysis, or interpretation of data: All authors

Drafting of the manuscript: All authors

Critical revision of the manuscript for important intellectual content: All authors

Statistical analysis: LE, JM and EL

Administrative technical, or material support. LE and EL

Supervision: JK, EL

\section{Acknowledgements}

We gratefully acknowledge the participating ICUs for their hard work in contributing data to the Swedish Intensive Care Registry.

\section{References}

1. Guérin C, Reignier J, Richard JC, Beuret P, Gacouin A, Boulain T, et al. Prone positioning in severe acute respiratory distress syndrome. N Engl J Med. 2013;368(23):2159-68.

2. Guérin C, Beuret P, Constantin JM, Bellani G, Garcia-Olivares P, Roca O, et al. A prospective international observational prevalence study on prone positioning of ARDS patients: the APRONET (ARDS Prone Position Network) study. Intensive Care Med. 2018;44(1):22-37.

3. Bellani G, Laffey JG, Pham T, Fan E, Brochard L, Esteban A, et al. Epidemiology, Patterns of Care, and Mortality for Patients With Acute Respiratory Distress Syndrome in Intensive Care Units in 50 Countries. Jama. 2016;315(8):788-800.

4. Ferrando C, Suarez-Sipmann F, Mellado-Artigas R, Hernández M, Gea A, Arruti E, et al. Clinical features, ventilatory management, and outcome of ARDS caused by COVID-19 are similar to other causes of ARDS. Intensive Care Med. 2020;46(12):2200-11. 
5. Clinical characteristics and day-90 outcomes of 4244 critically ill adults with COVID-19: a prospective cohort study. Intensive Care Med. 2021;47(1):60-73.

6. Camporota L, Sanderson B, Chiumello D, Terzi N, Argaud L, Rimmelé T, et al. Prone Position in Coronavirus Disease 2019 and Noncoronavirus Disease 2019 Acute Respiratory Distress Syndrome: An International Multicenter Observational Comparative Study. Critical care medicine. 2021.

7. Scaramuzzo G, Gamberini L, Tonetti T, Zani G, Ottaviani I, Mazzoli CA, et al. Sustained oxygenation improvement after first prone positioning is associated with liberation from mechanical ventilation and mortality in critically ill COVID-19 patients: a cohort study. Ann Intensive Care. 2021;11(1):63.

8. Albert RK, Keniston A, Baboi L, Ayzac L, Guérin C. Prone position-induced improvement in gas exchange does not predict improved survival in the acute respiratory distress syndrome. Am J Respir Crit Care Med. 2014;189(4):494-6.

9. Goligher EC, Ranieri VM, Slutsky AS. Is severe COVID-19 pneumonia a typical or atypical form of ARDS? And does it matter? Intensive Care Med. 2021;47(1):83-5.

10. Fan E, Del Sorbo L, Goligher EC, Hodgson CL, Munshi L, Walkey AJ, et al. An Official American Thoracic Society/European Society of Intensive Care Medicine/Society of Critical Care Medicine Clinical Practice Guideline: Mechanical Ventilation in Adult Patients with Acute Respiratory Distress Syndrome. Am J Respir Crit Care Med. 2017;195(9):1253-63.

11. Alhazzani W, Evans L, Alshamsi F, Møller MH, Ostermann M, Prescott HC, et al. Surviving Sepsis Campaign Guidelines on the Management of Adults With Coronavirus Disease 2019 (COVID-19) in the ICU: First Update. Critical care medicine. 2021;49(3):e219-e34.

12. Coppo A, Bellani G, Winterton D, Di Pierro M, Soria A, Faverio P, et al. Feasibility and physiological effects of prone positioning in non-intubated patients with acute respiratory failure due to COVID-19 (PRON-COVID): a prospective cohort study. Lancet Respir Med. 2020;8(8):765-74.

13. Langer T, Brioni M, Guzzardella A, Carlesso E, Cabrini L, Castelli G, et al. Prone position in intubated, mechanically ventilated patients with COVID-19: a multi-centric study of more than 1000 patients. Crit Care. 2021;25(1):128.

14. Mathews KS, Soh H, Shaefı S, Wang W, Bose S, Coca S, et al. Prone Positioning and Survival in Mechanically Ventilated Patients With Coronavirus Disease 2019-Related Respiratory Failure. Critical care medicine. 2021.

15. Stilma W, van Meenen DMP, Valk CMA, de Bruin H, Paulus F, Serpa Neto A, et al. Incidence and Practice of Early Prone Positioning in Invasively Ventilated COVID-19 Patients-Insights from the PRoVENT-COVID Observational Study. J Clin Med. 2021;10(20).

16. von Elm E, Altman DG, Egger M, Pocock SJ, Gøtzsche PC, Vandenbroucke JP. The Strengthening the Reporting of Observational Studies in Epidemiology (STROBE) statement: guidelines for reporting observational studies. J Clin Epidemiol. 2008;61(4):344-9.

17. Duggal A, Panitchote A, Siuba M, Krishnan S, Torbic H, Hastings A, et al. Implementation of Protocolized Care in ARDS Improves Outcomes. Respir Care. 2021;66(4):600-9. 
18. Chua EX, Zahir S, Ng KT, Teoh WY, Hasan MS, Ruslan SRB, et al. Effect of prone versus supine position in COVID-19 patients: A systematic review and meta-analysis. J Clin Anesth. 2021;74:110406.

19. Huang C, Soleimani J, Herasevich S, Pinevich Y, Pennington KM, Dong Y, et al. Clinical Characteristics, Treatment, and Outcomes of Critically III Patients With COVID-19: A Scoping Review. Mayo Clin Proc. 2021;96(1):183-202.

\section{Tables}

Table 1. Characteristics for patients with $\mathrm{PaO} 2 / \mathrm{FiO} 2 \leq 20 \mathrm{kPa}$ on invasive mechanical ventilation within 24 hours from ICU admission 


\section{All \\ Early prone $=$ NO \\ Early prone $=$ YES}

\section{Patient demographics, characteristics and comorbidities at ICU admission}

\begin{tabular}{llll} 
No. (\%) & 1714 & $1144(66.7)$ & $570(33.3)$ \\
\hline Women & $512(29.9)$ & $346(30.2)$ & $166(29.2)$ \\
\hline Age, median (IQR), y & $64(55-71)$ & $64(55-71)$ & $64(54-71)$ \\
\hline Age, Interval, y & & & \\
\hline$<40$ & $85(5)$ & $61(5.3)$ & $24(4.2)$ \\
\hline $40-49$ & $175(10.2)$ & $117(10.2)$ & $58(10.2)$ \\
\hline $50-59$ & $387(22.6)$ & $254(22.2)$ & $133(23.3)$ \\
$60-69$ & $549(32)$ & $362(31.6)$ & $187(32.8)$ \\
$70-79$ & $459(26.8)$ & $309(27)$ & $150(26.3)$ \\
$\geq 80$ & $59(3.4)$ & $41(3.6)$ & $18(3.2)$
\end{tabular}

Admission month

\begin{tabular}{llll} 
March - April 2020 & $514(30)$ & $426(37.2)$ & $88(15.4)$ \\
\hline May - August 2020 & $228(13.3)$ & $162(14.2)$ & $66(11.6)$ \\
\hline September - December 2020 & $326(19)$ & $203(17.7)$ & $123(21.6)$ \\
\hline January - April 2021 & $646(37.7)$ & $353(30.9)$ & $293(51.4)$
\end{tabular}

Location before ICU admission

\begin{tabular}{llll} 
Emergency department & $398(23.2)$ & $242(21.2)$ & $156(27.4)$ \\
\hline Hospital floor & $1316(76.8)$ & $902(78.8)$ & $414(72.6)$
\end{tabular}

Time from symptom to ICU admission, median (IQR), d

No. with data

Median (IQR), d

Hospital level

Tertiary
County
Local

Days at hospital before ICU admission

\begin{tabular}{lll}
1688 & 1124 & 564 \\
\hline $10(7-13)$ & $10(7-13)$ & $10(8-14)$
\end{tabular}

\begin{tabular}{lll}
$576(33.6)$ & $376(32.9)$ & $200(35.1)$ \\
\hline $964(56.2)$ & $636(55.5)$ & $328(57.5)$ \\
\hline $174(10.2)$ & $132(11.5)$ & $42(7.4)$ \\
\hline $2(0-4)$ & $2(0-4)$ & $2(0-5)$
\end{tabular}


Median (IQR), d

Pregnant, No.

16

14

2

Comorbidities

\begin{tabular}{llll} 
None & $493(28.8)$ & $356(31.1)$ & $137(24.0)$ \\
\hline One or more & $1221(71.2)$ & $788(68.9)$ & $433(76.0)$ \\
\hline Chronic hypertension & $825(48.1)$ & $521(45.5)$ & $304(53.3)$ \\
\hline Chronic cardiac disease & $239(13.9)$ & $162(14.2)$ & $77(13.5)$ \\
\hline COPD/Asthma & $275(16.0)$ & $178(15.6)$ & $97(17)$ \\
\hline Immune deficiency & $130(7.6)$ & $77(6.7)$ & $53(9.3)$ \\
\hline Chronic liver disease & $12(0.7)$ & $7(0.6)$ & $5(0.9)$ \\
\hline Chronic kidney disease & $97(5.7)$ & $69(6.0)$ & $28(4.9)$ \\
\hline Diabetes & $484(28.2)$ & $321(28.1)$ & $163(28.6)$ \\
\multicolumn{1}{c}{ Neuromuscular disease } & $18(1.1)$ & $10(0.9)$ & $8(1.4)$ \\
\hline Obesity $^{\mathrm{a}}$ & $188(11.0)$ & $111(9.7)$ & $77(13.5)$ \\
Malignancy $^{\mathrm{b}}$ & $31(1.8)$ & $21(1.8)$ & $10(1.8)$
\end{tabular}

Ventilatory parameters at ICU admission (within one hour before until one hour after admission)

\begin{tabular}{llll}
$\mathrm{PaO}_{2}, \mathrm{kPa}$. Median (IQR) & $8.5(7.4-9.8)$ & $8.6(7.4-9.9)$ & $8.3(7.3-9.4)$ \\
$\mathrm{FiO}_{2}, \%$. Median (IQR) & $80(70-100)$ & $80(65-95)$ & $85(70-100)$ \\
\hline $\mathrm{PaO}_{2} / \mathrm{FiO}_{2}$ ratio. Median (IQR) & $\begin{array}{l}11.0(8.5- \\
14.2)\end{array}$ & $\begin{array}{l}11.6(8.8- \\
14.8)\end{array}$ & $9.9(8.0-12.6)$
\end{tabular}

$\mathrm{PaO}_{2} / \mathrm{FiO}_{2}$ ratio categories. $\mathrm{kPa}$

$\begin{array}{llll}>13.3-\leq 26.6 & 525(30.6) & 415(36.3) & 110(19.3) \\ \leq 13.3 & 1189(69.4) & 729(63.7) & 460(80.7)\end{array}$

Treatment and vital signs at ICU admission (within one hour before until one hour after admission)

$\begin{array}{llll}\text { Vasopressor on admission, No (\%) } & 107(6.2) & 73(6.4) & 34(6.0)\end{array}$

Body temperature

\begin{tabular}{llll} 
No with data & 1647 & 1094 & 553 \\
\hline Degrees Celcius & $37.8(37.0-$ & $37.8(37.0-$ & $37.8(37.1-$ \\
& $38.4)$ & $38.5)$ & $38.3)$ \\
\hline Feverc, No (\%) & $602(36.6)$ & $423(38.6)$ & $180(32.5)$
\end{tabular}


Systolic blood pressure, min, $\mathrm{mmHg}$

No. with data

1669

1103

566

Median (IQR)

$119(95-135)$

$116(95-134)$

$120(100-139)$

Heart rate, maximum, beats/min

No. with data

1681

1116

565

Median (IQR)

$95(82-110)$

$95(80-110)$

$98(84-112)$

White blood cell count, $\times 10^{9} / \mathrm{L}$

No. with data

1638

1084

554

Median (IQR)

$9.3(6.8-$

12.9)

$9.0(6.5-12.6)$

$9.9(7.3-13.1)$

$\mathrm{pH}$

No. with data

1699

1130

569

Median (IQR)

$7.43(7.35-$

7.47)

$7.43(7.35-$

7.47)

7.44 (7.36-

7.48)

Creatinine, $\mathrm{mg} / \mathrm{dL}$

No. with data

1636

1085

551

Median (IQR)

72 (58-95)

71 (58-99)

$72(58-91)$

Bilirubin, $\mathrm{mg} / \mathrm{dL}$

No. with data

1596

1047

549

Median (IQR)

$9(6-12)$

$9(6-12)$

$9(6-12)$

SAPS3 at admission, median (IQR)

$58(53-65)$

$58(53-65)$

$58(53-66)$

Predicted risk of death (SAPS3), median (IQR), $\%$

$32(22-48)$

$32(22-46)$

$32(22-48)$

\section{Outcome}

30-day mortality

90-day mortality

$\begin{array}{ll}500(29.2) & 345(30.2) \\ 575(33.5) & 398(34.8)\end{array}$

$155(27.2)$

$177(31.1)$

Abbreviations: IQR, interquartile range; y: years; d: days; ICU, intensive care unit; COPD, chronic obstructive pulmonary disease; $\mathrm{PaO}_{2}$, arterial partial pressure of oxygen; $\mathrm{FiO}_{2}$, fraction of inspired oxygen; SAPS, simplified acute physiology score.

a Obesity is defined as $\mathrm{BMI}>40 \mathrm{~kg} / \mathrm{m}^{2}$ 
${ }^{b}$ Malignancy is defined as neoplasia spread beyond regional lymph nodes

${ }^{\mathrm{C}}$ Fever is defined as body temperature above $38^{\circ} \mathrm{C}$

Table 2. ICU care provided for patients in subcohort with low oxygenation and on early mechanical ventilation

\begin{tabular}{|llll|}
\hline Variable & No $(\%)$ & & \\
& All & $\begin{array}{l}\text { Early prone }= \\
\text { NO }\end{array}$ & $\begin{array}{l}\text { Early prone }= \\
\text { YES }\end{array}$ \\
& $(\mathbf{n = 1 7 1 4 )}$ & $\begin{array}{l}\mathbf{( n = 1 1 4 5 )} \\
(\mathbf{n}=\mathbf{5 6 9})\end{array}$ \\
\hline $\begin{array}{l}\text { Duration of invasive mechanical ventilation, } \\
\text { median (IQR) } h\end{array}$ & $\begin{array}{l}267(143- \\
450)\end{array}$ & $\begin{array}{l}265(137- \\
452)\end{array}$ & $269(165-444)$ \\
\hline Renal replacement therapy, No./total (\%) & $\begin{array}{l}312 / 1638 \\
(19.0)\end{array}$ & $\begin{array}{l}219 / 1082 \\
(20.2)\end{array}$ & $93 / 556(16.7)$ \\
\hline ECMO, No./total (\%) & $23 / 982(2.3)$ & $17 / 660(2.6)$ & $6 / 322(1.9)$ \\
\hline Tracheostomy, No. (\%) & $578(33.7)$ & $389(34.0)$ & $189(33.2)$ \\
\hline More than on admission ${ }^{\text {a }}$ & $576(33.6)$ & $408(35.7)$ & $168(29.5)$ \\
\hline ICU length of stay, median (IQR), d & $14(8-22)$ & $14(8-22)$ & $14(9-22)$ \\
\hline
\end{tabular}

Abbreviations: IQR, interquartile range; ECMO, extracorporeal membrane oxygenation; $h$, hours; ICU, intensive care unit; $d$, days

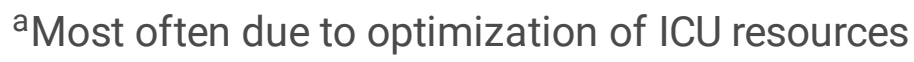

Table 3. Univariate and multivariable logistic regression analysis for 30-day mortality. 
Univariate

OR $(95 \% \mathrm{Cl})$
Multivariable

$P$ value $\quad$ OR $(95 \% \mathrm{Cl})$

$P$ value

Prone

Early $=$ NO

Reference

$0.86(0.69-1.08)$

0.2035

Early $=$ YES

Reference

Reference

Women

Men

$1.33(1.05-1.68)$

0.0183

$1.28(0.99$ - 1.67)

0.0645

Age, per year

$1.07(1.06-1.09) \quad<0.001$

$1.07(1.06-1.09)$

$<0.001$

Comorbidity

\begin{tabular}{|c|c|c|c|c|}
\hline Cardiac disease & $1.93(1.46-2.56)$ & $<0.001$ & $1.14(0.83-1.57)$ & 0.4203 \\
\hline COPD/Asthma & $1.08(0.81-1.43)$ & 0.5844 & $1.15(0.84-1.56)$ & 0.3846 \\
\hline Diabetes & $1.22(0.97-1.54)$ & 0.0807 & $1.12(0.86-1.45)$ & 0.4021 \\
\hline Obesity $^{a}$ & $0.63(0.43-0.89)$ & 0.0122 & $1.15(0.76-1.72)$ & 0.4979 \\
\hline Hypertension & $1.33(1.08-1.64)$ & 0.0071 & $0.86(0.67-1.10)$ & 0.2276 \\
\hline Immune deficiency & $1.52(1.04-2.19)$ & 0.0271 & $1.60(1.05-2.42)$ & 0.0279 \\
\hline Chronic liver disease & $0.81(0.18-2.72)$ & 0.7502 & $0.57(0.11-2.33)$ & 0.4637 \\
\hline Chronic kidney disease & $1.61(1.05-2.44)$ & 0.0268 & $1.05(0.65-1.66)$ & 0.8535 \\
\hline Neuromuscular disease & $0.69(0.20-1.94)$ & 0.5167 & $0.62(0.17-1.92)$ & 0.4384 \\
\hline Malignancy ${ }^{b}$ & $3.02(1.48-6.26)$ & 0.0025 & $2.12(0.97-4.69)$ & 0.0585 \\
\hline $\mathrm{PaO} 2 / \mathrm{FiO} 2$ ratio, per $1 \mathrm{kPa}$ increase & $0.97(0.94-1)$ & 0.0370 & $0.98(0.95-1.02)$ & 0.3362 \\
\hline SAPS3 ${ }^{\mathrm{C}}$, per 1 unit increase & $1.05(1.04-1.07)$ & $<0.001$ & $1.05(1.04-1.07)$ & $<0.001$ \\
\hline \multicolumn{5}{|l|}{ Admission months } \\
\hline March - April 2020 & Reference & & Reference & \\
\hline May - August 2020 & $0.57(0.39-0.83)$ & 0.0037 & $0.45(0.30-0.68$ & $<0.001$ \\
\hline September - December 2020 & $1.19(0.88-1.60)$ & 0.2489 & $0.80(0.57-1.11)$ & 0.1848 \\
\hline January - April 2021 & $0.91(0.71-1.18)$ & 0.4793 & $0.62(0.46-0.83)$ & 0.0014 \\
\hline
\end{tabular}


Abbreviations: OR, Odds Ratio; $\mathrm{Cl}$, Confidence Interval; COPD, chronic obstructive pulmonary disease; $\mathrm{PaO}_{2}$, arterial partial pressure of oxygen; $\mathrm{FiO}_{2}$, fraction of inspired oxygen; SAPS, simplified acute physiology score.

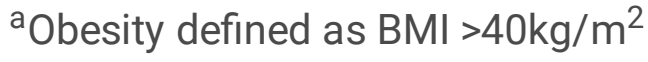

${ }^{b}$ Malignancy is defined as neoplasia spread beyond regional lymph nodes

${ }^{\mathrm{C}}$ Recalculated after excluding age, comorbidities and $\mathrm{PaO} 2 / \mathrm{FiO} 2$ ratio

aROC 0.749 for full model

Figures

7063 ICU patients with confirmed SARS-CoV-2 admitted between March 62020 and April 292021

574 ICU patients without COVID-

6489 ICU patients with COVID-

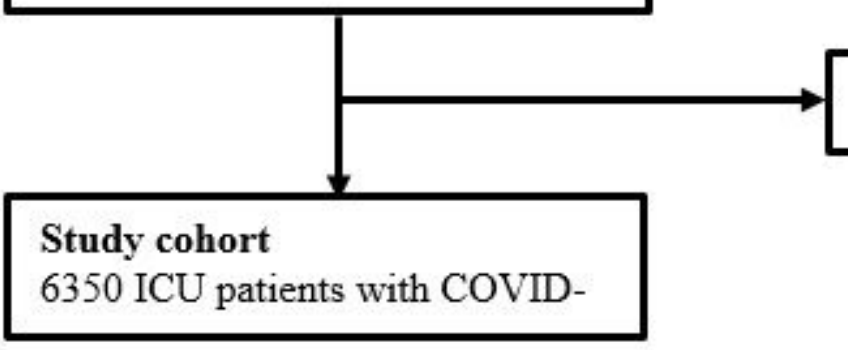

139 ICU patients without data on follow-up

Figure 1

Flow chart of included patients 
6350 ICU patients with COVID-

1978 patients with missing data on $\mathrm{PaO} 2 / \mathrm{FiO} 2$ at ICU admission

568 patients with $\mathrm{PaO} 2 / \mathrm{FiO} 2>20 \mathrm{kPa}$ at ICU

3804 ICU patients with COVID- 19 and $\mathrm{PaO} 2 / \mathrm{FiO} 2 \leq 20 \mathrm{kPa}$ at ICU

1877 ICU patients not receiving invasive mechanical ventilation within 24 hours from ICU admission

1927 ICU patients with COVID-19 receiving mechanical ventilation within 24 hours from ICU

$57 \mathrm{ICU}$ patients with no information on proning

1870 ICU patients with COVID-

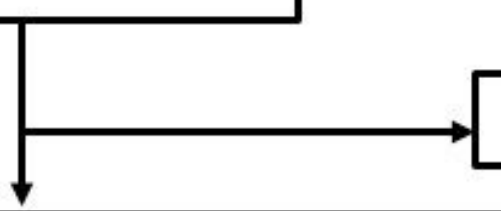

156 ICU patients with no information on time of start of proning

Included in final analysis

1714 ICU patients with COVID-19

- $\mathrm{PaO} 2 / \mathrm{FiO} 2 \leq 20 \mathrm{kPa}$ at ICU admission and

- Receiving invasive mechanical ventilation within 24 hours from ICU admission

Figure 2

Identification of patients with early mechanical ventilation and $\mathrm{PaO} 2 / \mathrm{FiO} 2 \leq 20 \mathrm{kPa}$ at ICU admission

\section{Supplementary Files}

This is a list of supplementary files associated with this preprint. Click to download.

- Supplementarytables.docx 